\title{
High pressure synthesis versus calcination - different approaches to crystallization of zirconium dioxide
}

\author{
Jarosław Kaszewski ${ }^{*}$, Sergiy Yatsunenko', Iwona Pełech², Ewa Mijowska², Urszula Narkiewicz ${ }^{2}$, \\ Marek Godlewski ${ }^{1}$ \\ ${ }^{1}$ Institute of Physics of the Polish Academy of Sciences, al. Lotników 32/46, 02-668 Warsaw, Poland \\ ${ }^{2}$ West Pomeranian University of Technology, Szczecin, Institute of Chemical and Environment Engineering, \\ ul. Pułaskiego 10, 70-322 Szczecin, Poland \\ *Corresponding author: e-mail: kaszewski@ifpan.edu.pl
}

\begin{abstract}
Calcination and microwave-assisted hydrothermal processing of precipitated zirconium dioxide are compared. Characterization of synthesized products of these two technologies is presented. The influence of thermal treatment up to $1200^{\circ} \mathrm{C}$ on the structural and spectroscopic properties of the so-obtained zirconium dioxide is examined. It was found that initial crystallization of material inhibits the crystal growth up to the $800^{\circ} \mathrm{C}$ (by means of XRD and TEM techniques), while the material crystallized from amorphous hydroxide precursor at $400^{\circ} \mathrm{C}$, exhibits $26 \mathrm{~nm}$ sized crystallites already. It was found using the TG technique that the temperature range $100-200^{\circ} \mathrm{C}$ during the calcination process is equivalent to a microwave hydrothermal process by means of water content. Mass loss is estimated to be about $18 \%$. Based on X-ray investigations it was found that the initial hydroxide precursor is amorphous, however, its luminescence activity suggests the close range ordering in a material.
\end{abstract}

Keywords: microwave, hydrothermal technology, zirconium dioxide, calcination.

\section{INTRODUCTION}

Hydrothermal technology is a widely applied method of ceramic materials synthesis ${ }^{1}$. It allows production of oxides, spinels and mixed oxides, as well as, metals in a crystalline form in the process excluding the calcination step ${ }^{2}$. Since the calcination severely modifies the structure of a product, hydrothermal conditions are considered as "mild". Hydrothermal technique exhibits a number of favorable properties, to mention a low energy consumption and a low environment pollution. Additionally, it allows to control some of the product properties, like grain shapes, crystallites size distribution and their nucleation, achieved via variation of the process conditions ${ }^{3}$. The high quality of the products of a hydrothermal synthesis origins from the way the material crystallizes in the subcritical/supercritical water environment. Water in hydrothermal conditions $\left(\mathrm{P}_{h}>100\right.$ $\mathrm{kPa}, \mathrm{T}_{\mathrm{h}}>25^{\circ} \mathrm{C}^{4}$ ) gains new physicochemical properties, like the decrease of viscosity, density and dielectric constant. It appears that transport properties in the reaction mixture are enhanced under the hydrothermal conditions, since the diffusion coefficient is inversely proportional to the viscosity of the medium ${ }^{4}$. The latter was revealed by the measurements of conductance in solutions under the hydrothermal conditions. These measurements confirmed that mobility of ions is higher than in ambient conditions and this effect compensates for the influence of a lowered dielectric constant in the overall solution conductance ${ }^{5}$. It implies one of the most important properties of the hydrothermal synthesis - the enhanced kinetics due to the efficient supply of reagents to the nucleation seeds. The properties of water change even more rapidly, when the critical point $\left(374.2^{\circ} \mathrm{C}, 22.1\right.$ $\mathrm{MPa}{ }^{6}$ ) is reached.

Many hydrothermal processing variations such as electrochemical hydrothermal ${ }^{9}$, mechanochemical hydrothermal ${ }^{10}$, sonochemical hydrothermal ${ }^{8}$, photochemical hydrothermal ${ }^{11}$, microwave hydrothermal ${ }^{7}$ are of importance in the development of technology. Also a number of techniques are combined with hydrothermal processing to reach the new properties of produced materials, to mention reactive electrode submerged arc process and biomineral templating synthesis ${ }^{12-15}$.

The new qualities appear in hydrothermal technology, when the heat is delivered to the reaction mixture by a microwave radiation. It allows the crystallization of high purity products, since the reaction mixtures do not have contact with the heating elements of the reactor ${ }^{7}$. Moreover, interaction of microwaves with the reagents in a solution causes a rapid chemical transformation. Thus, higher than in a classic hydrothermal process reaction rates are reached ${ }^{8}$, and a large number of nucleation points is created at the same time. It causes the concentration decrease in the feeding solution and reduces the growth of product crystallites. The resulting material is fine-grained and exhibits narrow crystallite size distribution ${ }^{16}$. Microwave heating opens also the possibility of obtaining new metastable phases in processed systems, since the local interactions with the reaction mixture create conditions far from the equilibrium. The properties of the obtained materials, like the size of crystals, morphology and agglomeration degree are controlled via the substrate quantitative ratio, $\mathrm{pH}$ and the temperature of the process ${ }^{17}$.

Zirconium dioxide is material of technological importance due to its superior properties like chemical stability, high melting point and high refractive index ${ }^{18} . \mathrm{ZrO}_{2}$ is also well known for its brilliant mechanical and heatproof properties. In consequence, $\mathrm{ZrO}_{2}$ found several applications in gas sensors, SOFCs and catalysts ${ }^{\mathbf{1 9}}$. High ionic conductivity allows $\mathrm{ZrO}_{2}$ applications in electro-ceramics. In addition, a wide energy band gap $\left(5-7 \mathrm{eV}^{\mathbf{2 0}}\right)$ allows potential applications as a visible light emitter ${ }^{21}$.

Many methods of zirconium dioxide synthesis are discussed in literature ${ }^{22-25}$, however calcination is one of the most used and described, considering its high accessibility and relative low complexity. Calcination is the process of heating solid state substrate below its melting point to 
induce chemical reaction or phase shift $\mathrm{t}^{26}$. The influence of process conditions in the zirconium dioxide calcination synthesis is widely discussed in literature, for instance in the references ${ }^{34,35}$. On the other hand, microwave hydrothermal production of zirconium dioxide is not commonly used, and only a limited number of papers is published on this topic, to mention ${ }^{27-29}$.

In the present work synthesis of zirconium dioxide using microwave hydrothermal technology is described and compared with the precipitation calcination method.

\section{EXPERIMENTAL}

\section{Material synthesis}

The investigated materials were prepared via precipitation calcination and microwave hydrothermal methods. The common step for both routes was preparation of the initial solution and precipitation steps. The solution was prepared by dissolving $22 \mathrm{~g}$ of $\mathrm{ZrO}\left(\mathrm{NO}_{3}\right)_{2} \cdot 6 \mathrm{H}_{2} \mathrm{O}$ (Aldrich) in $200 \mathrm{ml}$ of distilled water. Then, ammonia water solution (Chempur) was added to reach $\mathrm{pH}=10$. The resulting residue was then washed triply with distilled water to remove ammonium and nitrate $(\mathrm{V})$ ions. The residue was divided into two parts: the first was dried at $40^{\circ} \mathrm{C}$ by $24 \mathrm{~h}$, the second was introduced to microwave hydrothermal reactor. After drying the precursor was ground in the agate mortar and the resulting white powder was divided into 4 parts, three parts for different calcination temperatures and one left uncalcined. The calcination process was conducted at 400, 800 and $1200^{\circ} \mathrm{C}$ by 20 minutes in the air atmosphere. The wet part of the initial residue was processed in the teflon lined periodic microwave hydrothermal reactor (Ertec) operating at $2.45 \mathrm{GHz}$ with the power of $500 \mathrm{~W}$. The reaction mixture was prepared by combining the precipitate with distilled water with the volume ratio 1:1. The resulting suspension was homogenized by means of magnetic stirring and introduced into the teflon reaction vessel with the volume of $110 \mathrm{ml}$ to reach the fill coefficient of $80 \%$ volume. The vessel was closed in the steel coating of the reactor and the hydrothermal conditions were induced by constant irradiation of the reaction volume by microwaves. The interaction of the radiation with reaction mixture led to the increase of the temperature inside the vessel and in consequence autogenous pressure as the process was conducted in the closed space. The reaction volume was constantly heated until the pressure reached 5.5 MPa. Then the pressure was sustained for 20 minutes by microwave irradiation in the intervals of circa 3 minutes, to maintain its value in the range 5.4-5.6 MPa. The resulting powder was dried and divided into four parts, three parts for different calcination temperatures and one left uncalcined. Consequently, hydrothermal synthesized powders were also calcined at 400,800 and $1200^{\circ} \mathrm{C}$ by 20 minutes in air atmosphere.

\section{Instrumental analysis}

The X-ray diffraction (XRD) measurements were conducted using Philips X'Pert powder diffractometer operating with $\mathrm{Cu}_{\mathrm{Ka}}(0.154 \mathrm{~nm})$ radiation. The samples were pressed into the steel rings. Mean crystallite sizes
(MCS) were calculated using Scherrer's formula with the shape factor set to $\mathrm{k}=0.9$. Transmission electron microscopy (TEM) observations were conducted using FEI Tecnai F30 microscope. The samples were placed on the copper polycarbon coated grids 200 mesh (Agar). Powders were suspended in water and sonicated. Then, a drop of suspension was places on the grid, which was then hotplated at $40^{\circ} \mathrm{C}$ by 2 hours. Thermal analysis was conducted with TA Instruments SDT Q600 thermobalance. Ca. $20 \mathrm{mg}$ of the sample was placed in the corundum crucible and placed in the furnace, where measurements in the temperature range $20-1000^{\circ} \mathrm{C}$ were performed with the heating rate of $20^{\circ} \mathrm{C} / \mathrm{min}$. in the argon atmosphere. Luminescence measurements were conducted with CM2203 spectrofluorimeter. The source of excitation was $150 \mathrm{~W}$ xenon lamp. The instrument was equipped with R-928 Hamamatsu photomultiplier.

\section{RESULTS AND DISCUSSION}

\section{Structure}

Diffraction patterns of the obtained nanopowders are shown in Figure 1. The phase composition of samples changes in the function of calcination temperature. The powder resulting from microwave hydrothermal process is crystalline, which is indicated by a well resolved (101) reflex (at $2 \Theta \approx 30^{\circ}$ ) belonging to a tetragonal phase of $\mathrm{ZrO}_{2}$. Close to the same pattern weak monoclinic $\mathrm{ZrO}_{2}$ (111) and (i11) reflexes were detected, appearing as the shoulders of the $t(101)$ reflex. After annealing at 400 and $800^{\circ} \mathrm{C}$ phase composition does not change significantly, the relations in the intensities of particular XRD peaks are maintained. However, calcination at $1200^{\circ} \mathrm{C}$ results in the phase transformation to the monoclinic form.

On the other hand, the precipitation-calcination prepared powders show different structural behavior. The as-grown sample does not exhibit the long range ordering - diffraction reflexes are not observed. However, heating at the temperature as low as $400^{\circ} \mathrm{C}$ results in the appearance of sharp, well defined reflexes of the

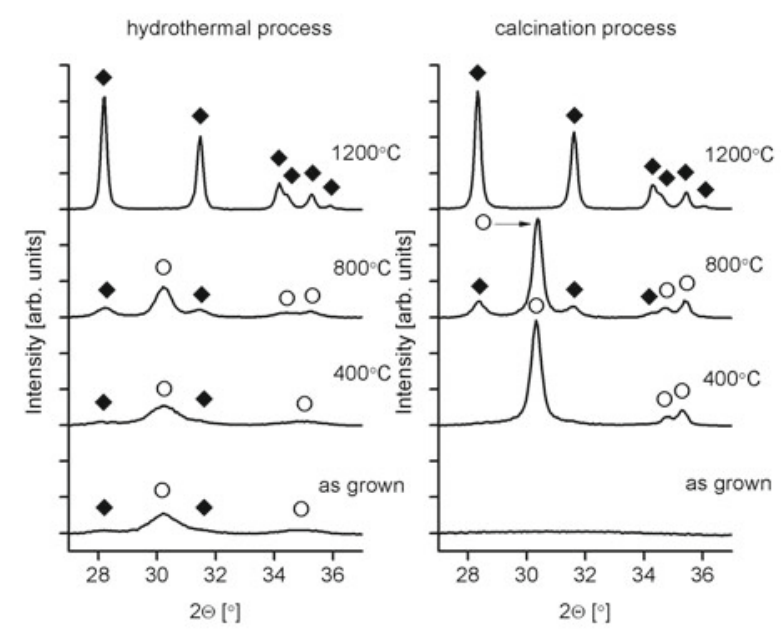

Figure 1. X-ray diffraction (XRD) patterns of the powders synthesized via microwave hydrothermal process and calcination. The samples were annealed at the temperatures shown on the picture. The phases are marked as: filled diamond - monoclinic $\mathrm{ZrO}_{2}$ phase, empty circle - tetragonal $\mathrm{ZrO}_{2}$ phase 
tetragonal $\mathrm{ZrO}_{2}$ phase. Next, heating at $800^{\circ} \mathrm{C}$ causes some amount of monoclinic phase to appear, however tetragonal phase is still dominant. Finally heating at $1200^{\circ} \mathrm{C}$ results in full transformation to the monoclinic $\mathrm{ZrO}_{2}$, in the same manner as for the microwave hydrothermal powder.

The explanation of such a behavior in both materials can be based on the knowledge of crystallization mechanism in applied technologies. In the case of microwave driven hydrothermal processing the nucleation process is induced by the interaction of high pressure and microwave radiation on hydroxide precursor in the presence of water. It was already proposed that the microwaves interact with the precursor locally, allowing the crystallization of small particles in the water environment ${ }^{30}$. The present research seems to confirm that. The heating does not change the structure of material up to the temperature at which the diffusion is efficient enough to make grain growth observable in the time as short as 20 minutes. Not surprisingly, the so-obtained material contains mainly crystalline zirconium oxide and its properties are mostly defined by the crystalline phase. Only at the highest temperature used in the present work the phase shift takes place. Then, the rate of grain growth increases and the critical size is exceeded, which is in general agreement with the size induced stabilization theory ${ }^{\mathbf{3 1}}$. The crystallites synthesized via microwave hydrothermal process are below critical size, thus the tetragonal $\mathrm{ZrO}_{2}$ phase is metastable at the room temperature (Fig. 2). For the as grown material MCS is ca. $6 \mathrm{~nm}$ for both $\mathrm{t}$ and $\mathrm{m}-\mathrm{ZrO}_{2}$ phases. The significant MCS growth occurs after heating at $800^{\circ} \mathrm{C}$ - to 10 and $12 \mathrm{~nm}$ for monoclinic and tetragonal phases, respectively. Calcination at $1200^{\circ} \mathrm{C}$ results in the MCS growth up to $78 \mathrm{~nm}-$ far above the critical size. The detail phase identification is, however, difficult considering very high FWHM values of the observed reflexes.

The cubic and tetragonal $\mathrm{ZrO}_{2}$ phases can be distinguished, in the range discussed, only by means of $\mathrm{t}(101)$ reflex shift or by observation of peak batch present at $2 \Theta \approx 35^{\circ}$. The broadening of reflexes is so high, that the resolution of the $\mathrm{t}(002)$ and $\mathrm{t}(110)$ (placed at $2 \Theta \approx 35^{\circ}$ ) is not possible. The size stabilization theory

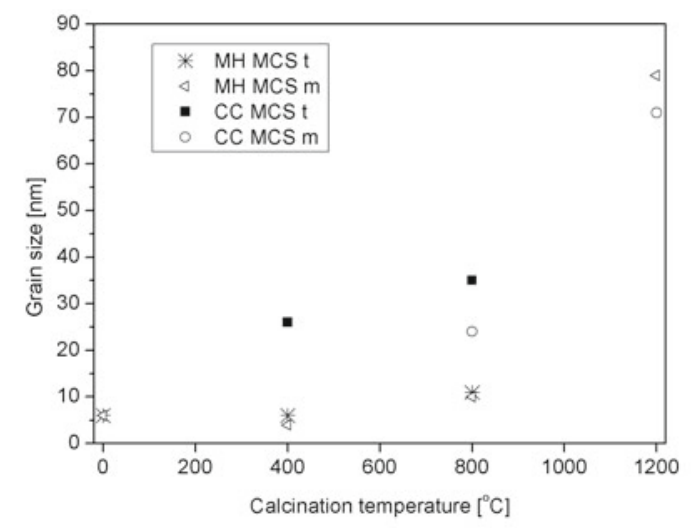

Figure 2. Mean crystallite sizes (MCS) calculated for the powders obtained by microwave hydrothermal and calcination methods in the function of annealing temperature. The calculations performed for tetragonal and monoclinic $\mathrm{ZrO}_{2}$ phases indicates, that in the grains of size below $10 \mathrm{~nm}$, cubic zirconia phase is present. However, after calcination at $800^{\circ} \mathrm{C}$, the tetragonal phase is clearly seen (Fig. 1). In the case of precipitation-calcination route, the precursor is amorphous, probably with the ordering typical for glasses $^{32}$. Heating of the amorphous precursor in the resistance furnace results in a temperature gradient in the reaction space. Thus the grains are less uniformly sized and higher quantity of crystallites may exceed the critical size. However, the phase purity in $400^{\circ} \mathrm{C}$ calcined sample suggests that the structure of the precursor has the significant impact on the resulting material phase composition.

The reaction mechanism depends also on the heat inertion of the processed material. In the absence of local interactions, the material crystallizes gradually, starting from the hydrated hydroxide precursor. The global character of grains growth results in a full crystallization already after heating at $400^{\circ} \mathrm{C}$ with the mean crystallite size of $26 \mathrm{~nm}$. Both large size and the presence of pure $\mathrm{t}-\mathrm{ZrO}_{2}$ phase is most likely related to the structure of the precursor itself. Similarly to the microwave hydrothermal process, the calcination removes the residual water from clusters containing $\mathrm{Zr}$ and $\mathrm{O}$. The monoclinic phase appears after calcination at $800^{\circ} \mathrm{C}$ with the MCS of 35 $\mathrm{nm}$. The different crystallization mechanisms are involved in both methods: in the case of microwave hydrothermal technology the surface interactions and size factor have significant contribution to the phase composition, whereas in the case of calcination technology - structure of precursor.

The transmission electron microscopy (TEM) images of hydrothermally and precipitation calcination prepared powders are shown in Figure 3. The as grown powder, as well as, amorphous precursor are shown in Figure $3 \mathrm{~A}$ and $3 \mathrm{~F}$. Additionally, the $10 \times 10 \mathrm{~nm}$ images of initial samples are shown in Fig. 3E and 3J for better understanding of local structure in materials prepared via both methods. The microwave hydrothermal sample has grainy structure with the sizes ranging from 4 to $12 \mathrm{~nm}$. The shape of crystallites is uniform, material is strongly agglomerated. The shapes are close to oval or round. The lattice fringes are seen, confirming crystalline structure of the particles. The precipitation prepared sample exhibits presence of large irregular structures with the sizes reaching hundreds of nanometers. These structures exhibit rugged texture. After calcination at $400^{\circ} \mathrm{C}$ the structures still remain as a main content of the sample. In microwave hydrothermal sample the decrease of inter-grain matter content is observed, thus the particles appear to be more separated from the others. It suggests, that the grains were coated with the hydroxide layer or inter-grain consisted of mainly hydroxide phase. Further calcination causes the sintering of crystallites, however the structures obtained via the precipitation-calcination method seem to be larger and less ordered in the nanometric scale. The annealing at $1200^{\circ} \mathrm{C}$ leads to the creation of strongly sintered agglomerates with the sizes of hundreds of nanometers, regardless the synthesis method used. 
A
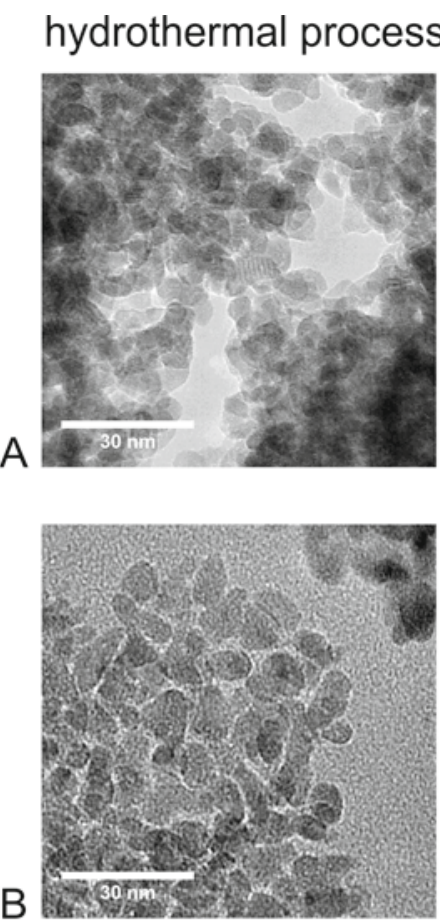

B

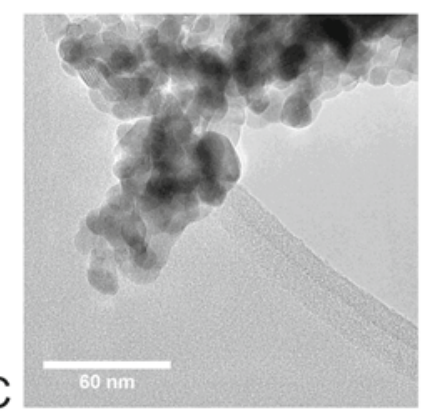

C

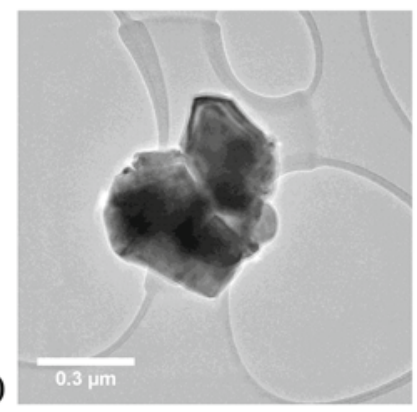

D

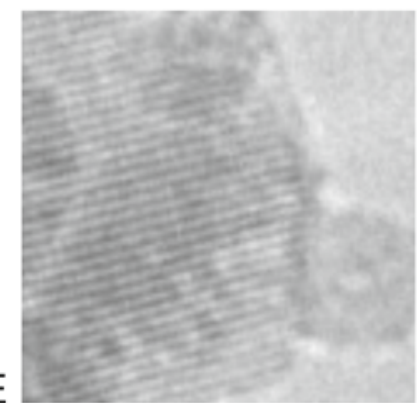

\section{calcination}
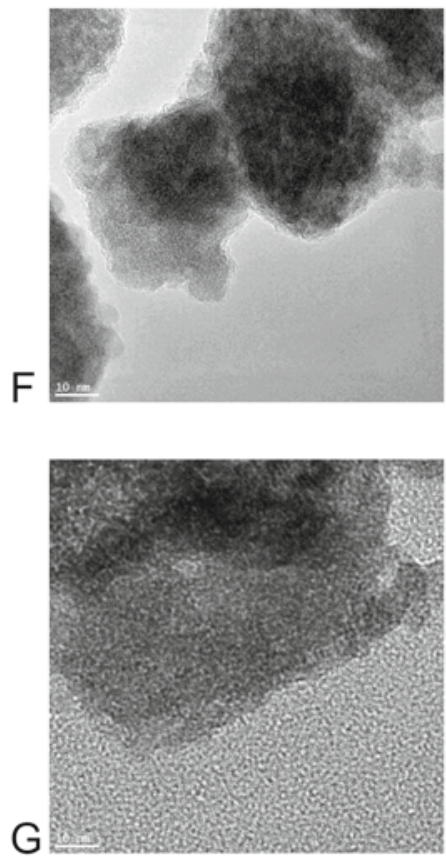

$400^{\circ} \mathrm{C}$

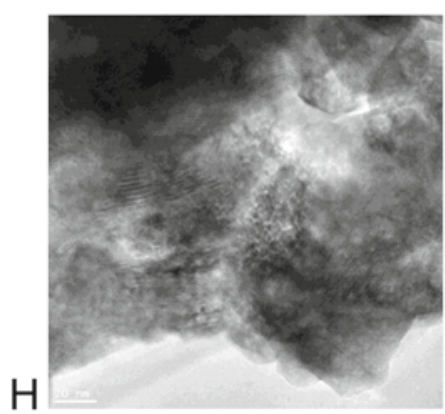

$800^{\circ} \mathrm{C}$

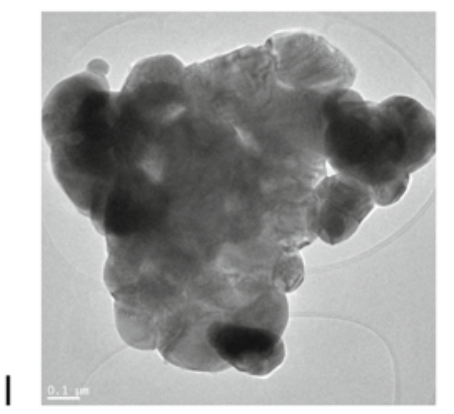

$1200^{\circ} \mathrm{C}$

as grown
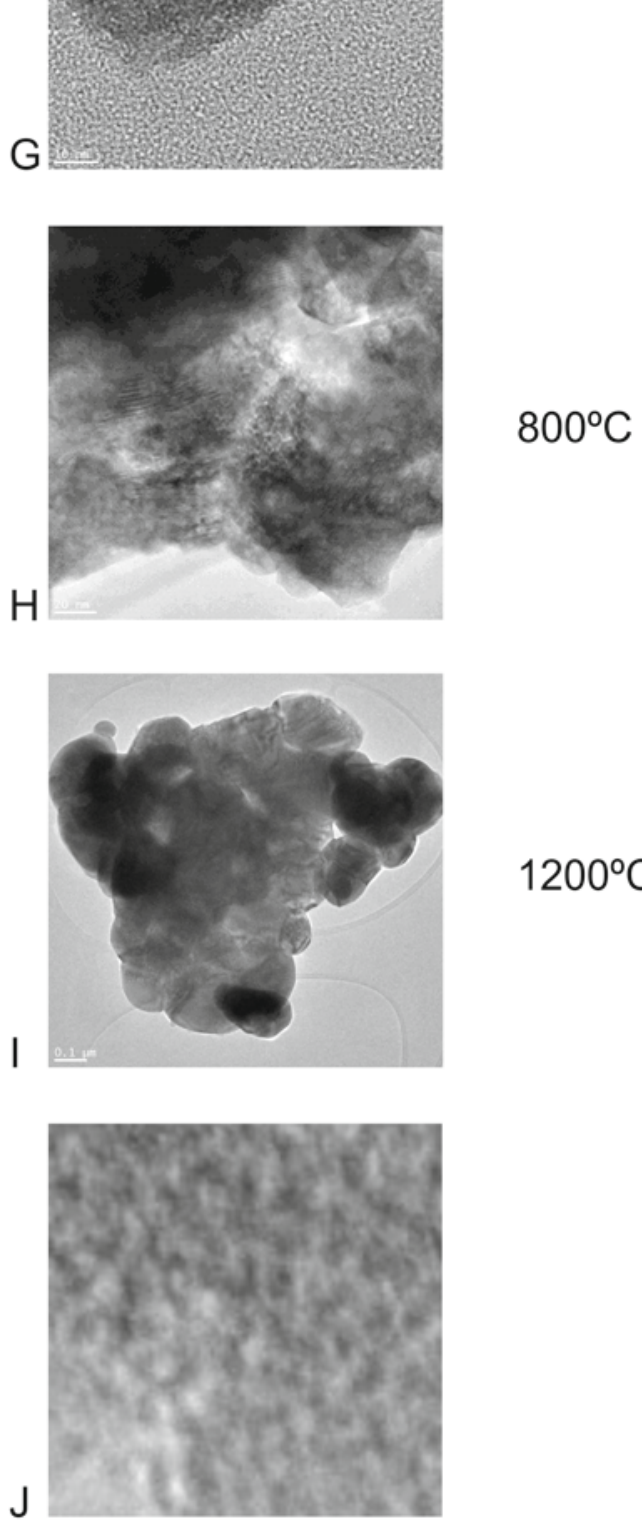

Figure 3. TEM micrographs revealing the evolution of powders morphology. The temperatures of annealing: $\mathrm{A}, \mathrm{F}-\mathrm{as}$ prepared; $\mathrm{B}, \mathrm{G}-400^{\circ} \mathrm{C} ; \mathrm{C}, \mathrm{H}-800^{\circ} \mathrm{C} ; \mathrm{D}, \mathrm{I}-1200^{\circ} \mathrm{C}$. The $10 \times 10 \mathrm{~nm}$ images of the as grown samples prepared via microwave hydrothermal and calcination processes are shown in figures $\mathrm{E}$ and $\mathrm{J}$ respectively

\section{Thermal behavior}

The thermogravimetric curves of the samples prepared both by precipitation and microwave hydrothermal process are shown in Fig 4ure The curves show diffe- rent overall mass loss in the temperature range of 20 to $1000^{\circ} \mathrm{C}$, as well as the shape. The mass losses are: microwave hydrothermal sample $-8 \%$, precipitation sample $-26 \%$. As the water is the main cause of the mass loss in the samples, we can conclude that the 


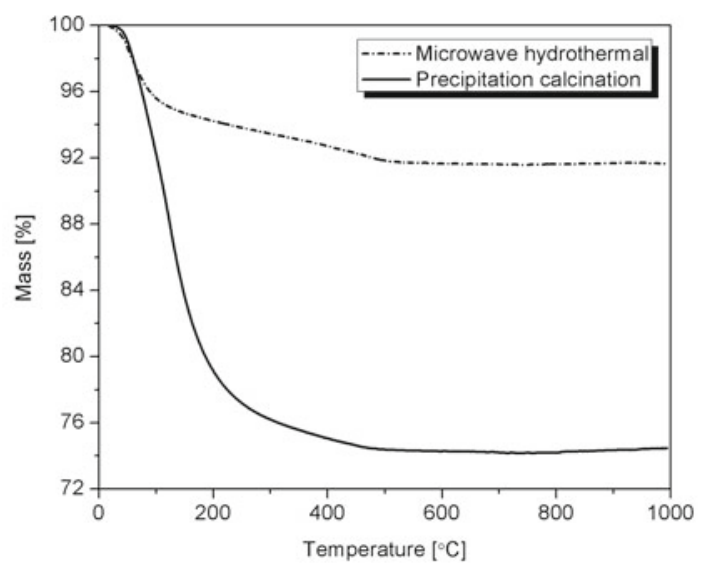

Figure 4. Thermogravimetric curves for the powders obtained via microwave hydrothermal processing (dotted line) and calcination (solid line)

crystalline phase content is higher in the microwave hydrothermal sample. The behavior up to ca. $100^{\circ} \mathrm{C}$ is the same for both samples, and is most likely related to the desorption of water from surface and pores of the material. Further character of the curves is associated with the decomposition of hydroxides and crystallization of zirconium dioxide. In the microwave hydrothermal prepared powder, after the evaporation step, the process related with the mass loss of ca. $4 \%$ takes place in the temperature range $100-500^{\circ} \mathrm{C}$. It is probably related to decomposition of zirconium hydroxide remnants or decomposition of surface hydroxide layer on the grains. Above $500^{\circ} \mathrm{C}$ the mass of sample does not change significantly. In the case of the amorphous precursor rapid mass loss takes place up to $200^{\circ} \mathrm{C}$ to ca. $78 \%$ of the initial mass. It suggests that decomposition process is associated with weakly bound water molecules up to $200^{\circ} \mathrm{C}$, then character of decomposition changes. Between 200 and $500^{\circ} \mathrm{C}$ mass loss is "mild" suggesting larger energies of decomposition, likely related to strongly bound crystallization water. The second step also finishes with ca. $4 \%$ of mass loss. The second step starts at the lower temperature in the sample synthesized via hydrothermal process, suggesting that the main dehydration reaction occurs in the high pressure step of synthesis. It appears that in the range $100-200^{\circ} \mathrm{C}$ the main dehydration step takes a place, which is equivalent of the dehydration during the hydrothermal reaction.

\section{Luminescence}

The photoluminescence (PL) spectra of the obtained nanopowders are shown in Figure 5. PL of the samples was excited in the UV region with the radiation wavelength of $260 \mathrm{~nm}(4.76 \mathrm{eV})$. The PL spectra consist of the wide emission band extending from 400 to $700 \mathrm{~nm}$. The intensity of the PL emission increases with the increase of the calcination temperature. The heating of microwave hydrothermal nanopowders was targeted to decrease the hydroxide content in material, as the hydroxyl groups are known of suppressing optical phenomena in solids. And as the product exhibits high crystallinity already after high pressure treatment, the short times of calcination were chosen to minimize the heating influence on the phase composition ${ }^{36}$. The PL maximum is placed at ca. $490 \mathrm{~nm}$ for all the powders. Observed PL spectra are asymmetric and the additional shoulder appears at ca. 620 $\mathrm{nm}$. The shape of the spectra is independent of heating
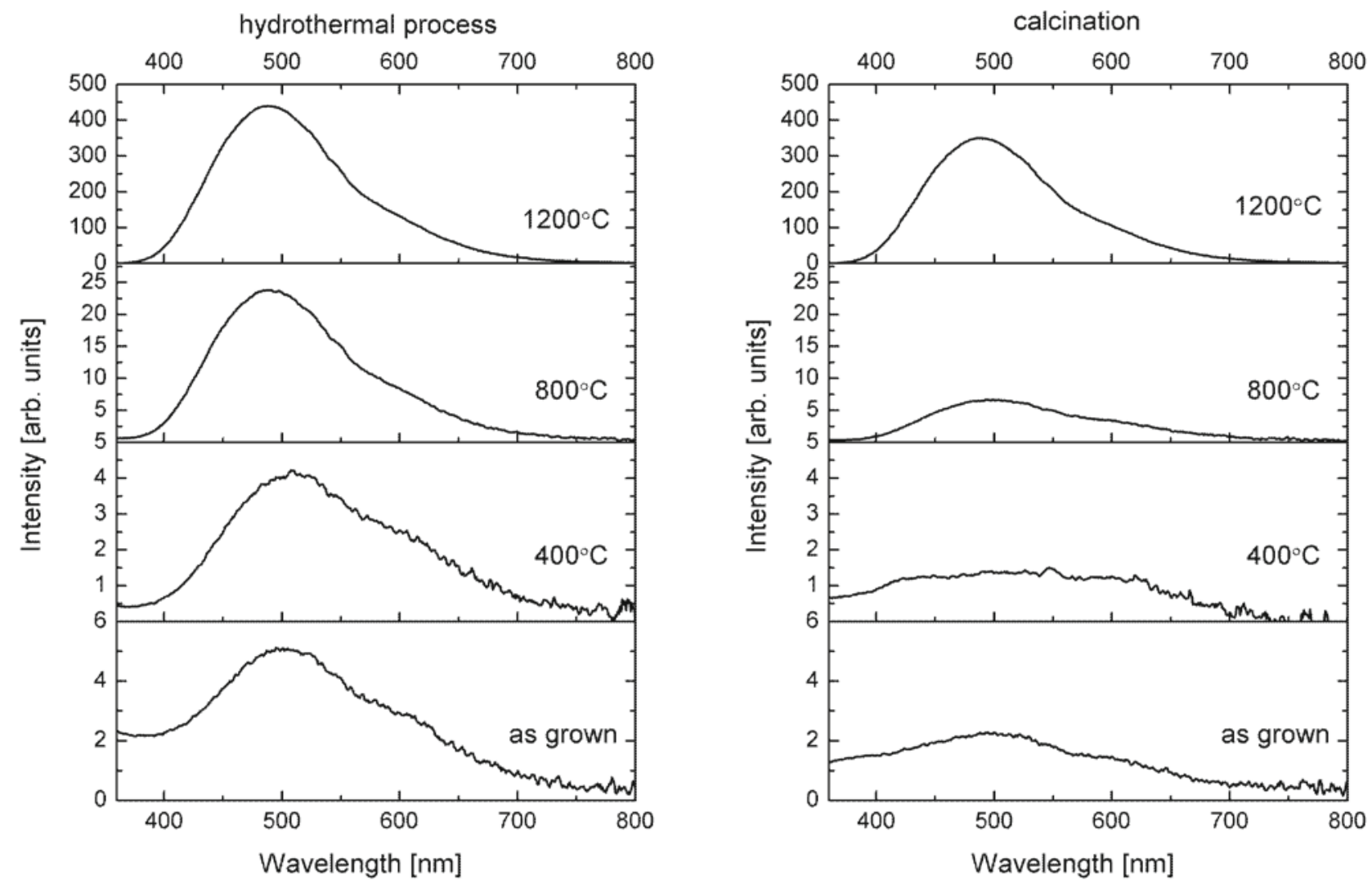

Figure 5. The luminescence emission spectra of samples prepared by microwave hydrothermal process (left) and calcination (right). The excitation wavelength was set to $260 \mathrm{~nm}$. The temperatures of annealing are shown on the pictures 
temperature in the microwave hydrothermal samples. This suggests that crystalline zirconium dioxide contain similar types and concentrations of centers responsible for the luminescence. The changes of intensity are related to phase composition and hydroxide content. Moreover, the increase of the PL intensity is associated with the variation of sizes of crystallites in the material. This suggests that the specific distribution of lattice defects is necessary to activate $\mathrm{ZrO}_{2}$ emission. In the precipitation-calcination prepared powders, the shape of spectra is influenced by heating temperature. However, the two band shape of PL spectra is preserved. The sample prepared at $1200^{\circ} \mathrm{C}$ exhibits very similar PL spectrum as the sample prepared via hydrothermal processing, suggesting, that the monoclinic phase is responsible for the bright emission. Additionally, the shape of the spectra observed in microwave hydrothermal prepared powders are very similar to the spectrum of the amorphous precursor, containing features at the same wavelengths. It proves that centers present in crystalline powders are also common in the precursor. Thus, are not related to a long range order present in a crystal lattice.

The absolute luminescence intensities are lower in the precipitation-calcination prepared samples, suggesting that the certain concentration and form of crystal lattice defects are responsible for the luminescent processes in a material, despite of the crystallite sizes differences. The band placed at $620 \mathrm{~nm}$ was also observed $\mathrm{in}^{\mathbf{3 3}}$, where appeared after annealing under mixture of gases containing $2 \%$ of oxygen and was associated with the creation of oxygen vacancies. Considering that we observe this band in both the microwave hydrothermal and calcination only prepared powders, even after annealing, we conclude, that $620 \mathrm{~nm}$ emission has the intrinsic character and is related to the existence of defects in oxygen sublattice.

\section{CONCLUSION}

The thermal growth of $\mathrm{ZrO}_{2}$ nanocrystals progresses in different ways, depending on the microwave hydrothermal treatment of initial hydroxide residue. The lower temperature of crystallization of the material starting from amorphous precursor was explained by a lower energy of hydroxide decomposition, in comparison with the energy needed to sinter grains of crystalline $\mathrm{ZrO}_{2}$. Despite its small size $(<10 \mathrm{~nm})$, the signs of sintering were observed exclusively after annealing at $800^{\circ} \mathrm{C}$. 3 steps of thermal decomposition were discriminated, with the step of massive water removing in the amorphous hydroxide sample between 100 and $200^{\circ} \mathrm{C}$. The mass of the removed water was estimated to $18 \%$ of overall sample mass, the step is not present in microwave hydrothermal prepared powder, suggesting that dehydration during high pressure process is its equivalent. However, after hydrothermal processing in the powder the strong bound hydroxyls are found, to finally decompose at ca. $500^{\circ} \mathrm{C}$. Thermal treatment also has changed the spectral properties of material. The increase of luminescence emission intensity is most likely caused by the presence of monoclinic $\mathrm{ZrO}_{2}$ phase. It was indicated by the increase of intensities in the samples containing monoclinic phase only, also characteristic spectral features are present in the powders containing low quantities of $\mathrm{m}-\mathrm{ZrO}_{2}$. On the other hand, the same emission bands are observed in amorphous precursor confirming its close range ordering. The microwave hydrothermal processing of zirconium dioxide based materials was found to bring new qualities relating to the typical precipitation calcination process. The properties of resulting material are more accurately size defined since the crystallites produced in microwave hydrothermal process are rapidly nucleated in all the reaction volume. The material is optically active in the visible region, indicating a possibility of application in the light emitting devices.

\section{LITERATURE CITED}

1. Byrappa, K. \& Adschiri, T. (2007). Hydrothermal technology for nanotechnology, Prog. Cryst. Growth Ch. 53, 117-166. DOI: $10.1016 /$ j.pcrysgrow.2007.04.001.

2. Zhu, X.H. \& Hang, Q.M. (2013). Microscopical and physical characterization of microwave and microwave-hydrothermal synthesis products, Micron 44, 21-44. DOI: 10.1016/j. micron.2012.06.005.

3. Riman, R.E., Suchanek, W.L. \& Lencka, M.M. (2002). Hydrothermal crystallization of ceramics, Ann. Chim. Sci. Mat. 27 (6), 15-36. DOI: 10.1016/S0151-9107(02)90012-7.

4. Byrappa, K. \& Yoshimura, M. (2000). Handbook of hydrothermal technology, William Andrew Publishing, Waltham, USA.

5. Franck, E.U. (1970). Water and aqueous solutions at high pressures and temperatures, Pure Appl. Chem. 24, 13-30. DOI: 10.1351/pac197024010013.

6. Franck, E.U. (1973). Properties of water, Int. Corros. Conf. Ser., 109-116.

7. Kornarneni, S., Li, Q., Stefansson, K.M. \& Roy, R. (1993). Microwave-hydrothermal processing for synthesis of electroceramic powders, J. Mater. Res. 8, 3176-3183. DOI: 10.1557/JMR.1993.3176.

8. Roy, R. (1994). Acceleration the kinetics of low-temperature inorganic syntheses, J. Solid State Chem. 111, 11-17. DOI:10.1006/jssc.1994.1192.

9. Switzer, J.A., Hung, C.J., Breyfogle, M., Shumsky, M.G., Vanleeuwen, R. \& Golden, T.D. (1994). Electrodeposited Defect Chemistry Superlattices, Science 264, 1573-1576. DOI: 10.1126/science.264.5165.1573.

10. Suchanek, W.L., Shuk, P., Byrappa, K., Riman, R.E., TenHuisen, K.S. \& Janas, V.F. (2002). Mechanochemical-hydrothermal synthesis of carbonated apatite powders at room temperature, Biomaterials 23, 699-710. DOI: 10.1016/S01429612(01)00158-2.

11. Puippe, J.C., Acosta, R.E. \& von Gutfeld, R.J. (1981). Investigation of laser enhanced electroplating mechanisms, J. Electrochem. Soc. 128, 2539-2545. DOI: 10.1149/1.2127287.

12. Kumar, A. \& Roy., R. (1988). RESA-A wholly new process for fine oxide powder preparation, J. Mater. Res. 3(6), 1373-1377. DOI: 10.1557/JMR.1988.1373.

13. Ehrlich, H., Simon, P., Motylenko, M., Wysokowski, M., Bazhenov, V.V., Galli, R., Stelling, A.L., Stawski, D., Ilan, M., Stöcker, H., Abendroth, B., Born, R., Jesionowski, T., Kurzydłowski, K.J. \& Meyer, D.C. (2013). Extreme Biomimetics: formation of zirconium dioxide nanophase using chitinous scaffolds under hydrothermal conditions, J. Mater. Chem. B 2013, 1, 5092-5099. DOI: 10.1039/C3TB20676A.

14. Wysokowski, M., Motylenko, M., Bazhenov, V.V., Stawski, D., Petrenko, I., Ehrlich, A., Behm, T., Kljajic, Z., Stelling, A.L., Jesionowski, T. \& Ehrlich, H. (2013). Poriferan chitin as a template for hydrothermal zirconia deposition, Front. Mater. Sci. 7(3), 248-260. DOI:10.1007/s11706-013-0212-x.

15. Wysokowski, M., Motylenko, M., Stöcker, H., Bazhenov, V.V., Langer, E., Dobrowolska, A., Czaczyk, K., Galli, R., Stelling, A.L., Behm, T., Klapiszewski, Ł., Ambrożewicz, D., Nowacka, M., Molodtsov, S.L., Abendroth, B., Meyer, D.C., 
Kurzydłowski, K.J., Jesionowski, T. \& Ehrlich, H. (2013). An extreme biomimetic approach: hydrothermal synthesis of $\beta$-chitin/ZnO nanostructured composites, J. Mater. Chem. B 1, 6469-6476. DOI: 10.1039/C3TB21186J.

16. Opalińska, A., Pielaszek, R., Łojkowski, W., Leonelli, C., Matysiak, H., Wejrzanowski, T. \& Kurzydłowski, K.J. (2010). Grain size and grain size distribution of Pr-doped zirconia nanopowders determined by different methods, Materiały Ceramiczne 62, 550-555.

17. Komarneni, S., Hussein, M.Z., Liu, C., Breval, E. \& Malla, P.B. (1995). Microwave-hydrothermal processing of metal clusters supported in and/or on montmorillonite, Eur. J. Solid State Inorg. Chem. 32, 837-849.

18. Lin, C., Zhang, C. \& Lin, J. (2007). Phase transformation and photoluminescence properties of nanocrystalline $\mathrm{ZrO}_{2}$ powders prepared via the Pechini-type sol-gel process, J. Phys. Chem. C 111, 3300-3307. DOI: 10.1021/jp0666151.

19. Sridhar, K.R. \& Blanchard, J.A. (1999). Electronic conduction in low oxygen partial pressure measurements using an amperometric zirconia oxygen sensor, Sensor. Actuator. B-Chem. 59, 60-67. DOI:10.1016/S0925-4005(99)00233-6.

20. French, R.H., Glass, S.J., Ohuchi, F.S., Xu, Y.N. \& Ching, W.Y. (1994). Experimental and theoretical determination of the electronic structure and optical properties of three phases of $\mathrm{ZrO}_{2}$, Phys. Rev. B 49 (8), 5133-5142. DOI:10.1103/ PhysRevB.49.5133.

21. Li, Q., Ai, D., Dai, X. \& Wang, J. (2003). Photoluminescence of nanometer zirconia powders, Powder Technol. 137, 34-40. DOI: 10.1016/j.powtec.2003.08.028.

22. Feng, Z., Postula, W.S., Akgerman, A. \& Anthony, R.G. (1995). Characterization of zirconia-based catalysts prepared by precipitation, calcination and modified sol-gel methods, Ind. Eng. Chem. Res. 34, 78-82. DOI: 10.1021/ie00040a005.

23. Somiya, S. \& Akiba, T. (1999). Hydrothermal zirconia powders: A bibliography, J. Eur. Ceram. Soc. 19, 81-87. DOI: 10.1016/S0955-2219(98)00110-1.

24. Amberg, M. \& Gunter, J.R. (1996). Metastable cubic and tetragonal zirconium dioxide, prepared by thermal oxidation of the dichalcogenides, Solid State Ionics 84, 313-321. DOI: 10.1016/0167-2738(96)00020-3.

25. Kaddouri, A., Mazzocchia, C., Tempesti, E. \& Anouchinsky, R. (1998). On the activity of $\mathrm{ZrO}_{2}$ prepared by different methods, J. Therm. Anal. 53, 97-109. DOI: 10.1023/A:1010110024557.

26. McNaught, A. D. \& Wilkinson, A. (1997). IUPAC Compendium of chemical terminology, 2nd ed. Blackwell Scientific Publications, Oxford.

27. Kornarneni, S., Roy, R. \& Li, Q.H. (1992). Microwave-hydrothermal synthesis of ceramic powders, Mat. Res. Bull. 27, 1393-1405. DOI: 10.1016/0025-5408(92)90004-J.

28. Bondioli, F., Leonelli, C., Manfredini, T., Ferrari, A.M., Caracoche, M.C., Rivas, P.C. \& Rodriguez, A.M. (2005). Microwave-hydrothermal synthesis and hyperfine characterization of praseodymium-doped nanometric zirconia powders, J. Am. Ceram. Soc. 88 (3), 633-638. DOI: 10.1111/j.1551-2916.2005.00093.x.

29. Smits, K., Grigorjeva, L., Millers, D., Sarakovskis, A., Opalinska, A., Fidelus, J.D. \& Łojkowski, W. (2010). Europium doped zirconia luminescence, Opt. Mater. 32, 827-831. DOI: 10.1016/j.optmat.2010.03.002.

30. Mingos, D.M.P. (1994). The applications of microwaves in chemical syntheses, Res. Chem. Intermed. 20, 85-91. DOI: 10.1163/156856794X00090.

31. Garvie, R.C. (1978). Stabilization of the tetragonal structure in zirconia microcrystals, J. Phys. Chem. 82 (2), 218-224. DOI: $10.1021 / \mathrm{j} 100491 \mathrm{a} 016$.

32. Mondal, A. \& Ram, S. (2004). Reconstructive phase formation of $\mathrm{ZrO}_{2}$ nanoparticles in a new orthorhombic crystal structure from an energized porous $\mathrm{ZrO}(\mathrm{OH})_{2} \cdot \mathrm{xH}_{2} \mathrm{O}$ precursor, Ceram. Int. 30, 239-249. DOI: 10.1016/S0272-8842(03)00095-6.
33. Smits, K., Grigorjeva, L., Millers, D., Sarakovskis, A., Grabis, J. \& Łojkowski, W. (2011). Intrinsic defect related luminescence in $\mathrm{ZrO}_{2}$, J. Lumin. 131, 2058-2062. DOI: 10.1016/j. jlumin.2011.05.018.

34. Guo, G.Y., Chen, Y.L. \& Ying, W.J. (2004). Thermal, spectroscopic and $\mathrm{X}$-ray diffractional analyses of zirconium hydroxides precipitated at low $\mathrm{pH}$ values, Mater. Chem. Phys. 84, 308-314. DOI: 10.1016/j.matchemphys.2003.10.006.

35. Zhang, Y.L., Jin, X.J., Rong, Y.H., Hsu, T.Y., Jiang, D.Y. \& Shi, J.L. (2006). The size dependence of structural stability in nano-sized $\mathrm{ZrO}_{2}$ particles, Mater. Sci. Eng. A 438-440, 399-402. DOI: 10.1016/j.msea.2006.03.109.

36. Glushkova, V.B. \& Lapshin, A.N. (2003). Specific features in the behavior of amorphous zirconium hydroxide: I. Sol-gel processes in the synthesis of zirconia, Glass Phys. Chem. 29, 415-421. DOI: 10.1023/A:1025137313344. 